coverage. With the help of additive technologies, 3D-printed applicators can be tailored to the patient's tumour and anatomy.

Methodology In this report two cases of gynaecological cancer, one vaginal and one recurrent endometrial cancer are presented. The design of the applicator was based on MRI images of the patient with the standard Varian vaginal cylinder inserted. Parallel and oblique needles were virtually added to the planning system to get the best possible coverage of the tumour while respecting the dose constraints to the organs at risk (OARs). Individual applicators were made from biocompatible polyamide PA 12 with selective laser sintering (SLS) technology (figure 1). The next BT was performed with an individual applicator in situ. Rectal ultrasound was used for needle guidance. The dose-volume histogram (DVH) parameters for each patient were compared according to the planning aims. The planning aim for D90 high-risk clinical target volume (CTV-THR) was to reach physical dose > 20.5 Gy per one BT fraction delivered in 24 pulses of pulsed dose rate (PDR) BT.

Results The DVH parameters for both cases per one BT fraction are presented in table 1 . The procedure and the implantation of the needles was performed without complications in regional anaesthesia. The applicator was well tolerated, no adverse effect was reported during the treatment or removal of the applicator.

Conclusion The advantages of using an individually-designed multi-channel vaginal applicator are:

- better target coverage in advanced tumours extended in the vagina

- can be used in a narrow vagina

- implantation guidance of several oblique and parallel needles can be performed with minimized trauma to the surrounding tissue

- allows for the treatment of several tumour locations in the same BT fraction

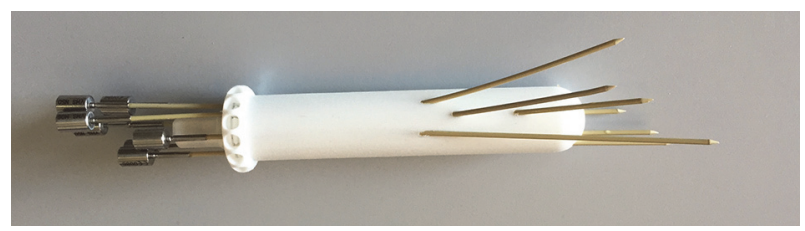

Abstract 86 Figure 1 Multi-channel vaginal applicator with oblique and parallel needles

Abstract 86 Table 1 The dose-volume histogram parameters for both cases per one pulse-dose rate brachytherapy fraction

\begin{tabular}{|c|c|c|c|}
\hline DVH parameter & Patient 1 & Patient 2 & Planning aims* \\
\hline CTV-T $_{\mathrm{HR}}$ D98 & $18.7 \mathrm{~Gy}$ & $17.4 \mathrm{~Gy}$ & $>14.8 \mathrm{~Gy}$ \\
\hline CTV-T $_{\mathrm{HR}}$ D90 & $23.4 \mathrm{~Gy}$ & $21.5 \mathrm{~Gy}$ & $>20.5 \mathrm{~Gy}$ \\
\hline CTV-T $\mathrm{HR}_{\mathrm{R}} \mathrm{V100}$ & $19.3 \mathrm{~Gy}$ & $18.9 \mathrm{~Gy}$ & $>18.0 \mathrm{~Gy}$ \\
\hline Bladder $D_{2 \mathrm{~cm}^{3}}$ & $13.3 \mathrm{~Gy}$ & $12.5 \mathrm{~Gy}$ & $14.6 \mathrm{~Gy}$ \\
\hline Rectum $D_{2 \mathrm{~cm}^{3}}$ & $9.5 \mathrm{~Gy}$ & $10.8 \mathrm{~Gy}$ & $11.2 \mathrm{~Gy}$ \\
\hline Sigmoid $D_{2 \mathrm{~cm}^{3}}$ & $5.7 \mathrm{~Gy}$ & $5.7 \mathrm{~Gy}$ & $13.0 \mathrm{~Gy}$ \\
\hline Bowel $D_{2 \mathrm{~cm}^{3}}$ & $5.6 \mathrm{~Gy}$ & $9.5 \mathrm{~Gy}$ & $13.0 \mathrm{~Gy}$ \\
\hline \multicolumn{4}{|c|}{$\begin{array}{l}\text { *The planning aims are physical doses calculated per one BT fraction according to Embrace II } \\
\text { study. } \\
\text { BT fraction was delivered in } 24 \text { pulses using pulsed dose rate BT, one pulse per hour, repair }\end{array}$} \\
\hline
\end{tabular}

Disclosures Helena Barbara Zobec Logar, Robert Hudej and Manja Kobav have nothing to disclose.

\section{PROGNOSTIC FACTORS RELATED TO RECURRENCE AND SURVIVAL OF EARLY STAGE VULVAR SQUAMOUS CELL CARCINOMA}

${ }^{1}$ Yun Wang, ${ }^{2}$ Kristina Lindemann, ${ }^{3}$ Kjersti Bruheim, ${ }^{3}$ Anne Barrameda, ${ }^{4}$ Ben Davidson, ${ }^{3}$ Tone Skeie-Jensen. 'Department of Gynecologic Oncology, Oslo University Hospital-Radium Hospital, Oslo, Norway; ${ }^{2}$ Department of Gynecologic Oncology, Oslo University HospitalRadium Hospital, Oslo, Norway; Faculty of Medicine, Institute of Clinical Medicine, University of Oslo, Oslo, Norway; ${ }^{3}$ Department of Oncology, Oslo University HospitalRadium Hospital, Oslo, Norway; ${ }^{4}$ Department of Pathology, Oslo University Hospital-Radium Hospital, Oslo, Norway; Faculty of Medicine, Institute of Clinical Medicine, University of Oslo, Oslo, Norway

\subsection{6/ijgc-2020-ESG0.179}

Introduction/Background In patients treated for clinical early stage vulvar squamous cell carcinoma (VSCC), recurrence rate is reported to be up to $37 \%$. Identification of prognostic factors related to recurrence and survival will help to tailor surgical treatment and follow-up with the aim to prevent recurrence and prolong survival. The aim of the study is to evaluate prognostic factors for recurrence and survival among patients with early stage VSCC.

Methodology This is a retrospective study of patients with early stage VSCC who were treated at Oslo University Hospital - Radium hospital between 01.01.2006 and 31.12.2016. Clinicopathological characteristics, treatment and follow-up were extracted from the medical records. Univariate analysis and multivariate logistic regression and Cox proportional hazard regression analysis were used to identify prognostic factors for recurrence, time to recurrence (TTR) and overall survival (OS). A p-value of $<0.05$ was considered to be statistically significant.

Results 133 patients with clinical stage I VSCC were included. All patients underwent primary vulva surgery and evaluation of groin lymph node status. The median age was 64 years (range 29-93 years), and groin lymph node metastases were identified in $22.6 \%$ of patients. The median follow-up time was 67 months (range 5-165). The 5-year recurrence and survival rates were $23.3 \%$ (31 of 133) and 72.2\% (96 of 133), respectively. In multivariate analysis, the presence of lichen sclerosus and groin lymph node metastasis were independent significant prognostic factors for recurrence and TTR, with an odds ratio (OR) $(95 \% \mathrm{CI})$ of $5.37(2.13-13.53)$ and 2.8 (1.17-6.72), respectively, for recurrence, and a HR (95\% CI) of $2.6(1.35-5.19)$ and $2.2(1.13-4.26)$ for TTR, respectively. Age $>70$ years and a history of recurrence were independent prognostic factors for OS, with an HR (95\% CI) of 3.0 (1.58-5.65) and 2.1 (1.57-6.15), respectively. Pathological tumor-free margin distance was not an independent prognostic factor for recurrence, TTR or OS, neither using a cutoff of 8 millimeters, nor as a continuous variable.

Conclusion Patients with lichen sclerosus and groin lymph node metastasis have a higher risk for recurrence and shorter TTR. Patients with age $>70$ years and a history of recurrence have significantly poorer OS. Pathological tumor-free margin distance was not an independent prognostic factor for recurrence and survival.

Disclosures All authors declare no conflict of interest. 